\title{
Regression effect in judgments to determine equal response contours
}

\author{
WILLIAM E. KAPPAUF \\ University of Illinois, Champaign, Illinois 61802
}

\begin{abstract}
The data of Sheldon's experiment (1973) on equal onset contours for vibrotactile stimuli display a marked regression effect. This effect is one of bias and is not to be confused with statistical regression toward the mean. It resembles the effect found by Stevens $(1955,1961)$ and others in data from scale-matching experiments, but the present effect involves bias toward the level of the standard on the dimension which the subject is adjusting to achieve his match, rather than being related to the entire stimulus range. Both regression effects appear to increase with the difficulty of the matching task.
\end{abstract}

It appears to have been Stevens (1955) who first drew attention to the fact that subjects who are required to match a tone and a noise for loudness at a series of different intensity levels arrive at two different functions relating the intensities of the matched sounds when, on the one hand, they adjust the tone to match the noise and, on the other, they adjust the noise to match the tone. In a subsequent paper, he and Greenbaum (1966) substantiated the position that this linding generalizes to other perceptual dimensions and may be expected to apply not only in cases where the matching relation between two scales is investigated by the method of adjustment, but also in other judgment situations where correspondences or equalities can be established in either of two directions. The typical result is that the slope of the psychophysical function that is obtained depends upon which of the stimuli in the matching situation is under the subject's control, and that when the subject controls one and then the other, the two functions intersect. The effect portrayed by these intersecting functions has been associated with the "central tendency" in judgment (after Hollingworth, 1910): in his response, the subject uses too narrow a range on the scale of the adjusted variable, and his matching settings thus turn out to be distributed nearer the center of that scale than expected. Stevens and Greenbaum (1966) have called it a regression effect. a name readily suggested by the graphic similarity of the two intersecting functions to the intersection of regression lines on a scatterplot.

A particularly striking example of an effect like this is to be found in the results of the experiment on equal-onset contours for vibrotactile stimuli recently reported in this journal by Sheldon (1973). It is the purpose of the present note to discuss Sheldon's results and what they imply for potential bias and method in other experiments on equal response contours.

Reprints may be obtained by addressing the author at the Department of Psychology. University of Illinois. Champaign. Illinoir 61820 .

\section{SHELDON'S DATA ON EQUAL ONSET CONTOURS}

Sheldon ${ }^{1}$ has kindly provided the writer with transcripts of some of his original data, and from these his results have been replotted in the form shown in Figure 1, where the magnitude of the effect of the direction of adjustment on the slopes of the regression lines becomes clear.

Sheldon's subjects were instructed to observe the cutaneous "surge" or abruptness of onset which occurs when a vibrotactile stimulus is delivered to the ball of the index finger. This cutaneous surge was known from earlier work to depend both on the rise time of the stimulus and on the intensity or final amplitude of the stimulus, and Sheldon therefore undertook a determination of equal-surge contours over several portions of the intensity:rise-time domain. Four subjects, the same subjects, served in each of the two experiments. Their task, in each case, was to match the surge of a variable stimulus to the surge of the standard stimulus. In the first experiment, the experimenter set the intensity level of the variable stimulus and the subject adjusted its rise time until he judged variable and standard to be alike in surge. In the second, the experimenter set the rise time of the variable stimulus and the subject adjusted its intensity until the equality judgment was made. These may be called the method of rise-time adjustment and the method of intensity adjustment, respectively.

Sheldon used four standards that were common to the two experiments: stimuli of (a) $40 \mathrm{~dB}$ intensity above the subject's threshold, with a rise time of $100 \mathrm{msec}$ (b) $40 \mathrm{~dB}$ with a rise time of $200 \mathrm{msec}$, (c) $40 \mathrm{~dB}$ with a rise time of $500 \mathrm{msec}$, and (d) $40 \mathrm{~dB}$ with a rise time of $1.000 \mathrm{msec}$. The final data consisted of tive intensity:rise-time combinations that were judged equal in surge to each of these standards by each of the foregoing methods. establishing eight different empirical equal-onset or equal-surge contours. Sheldon plotted his data separately for the 

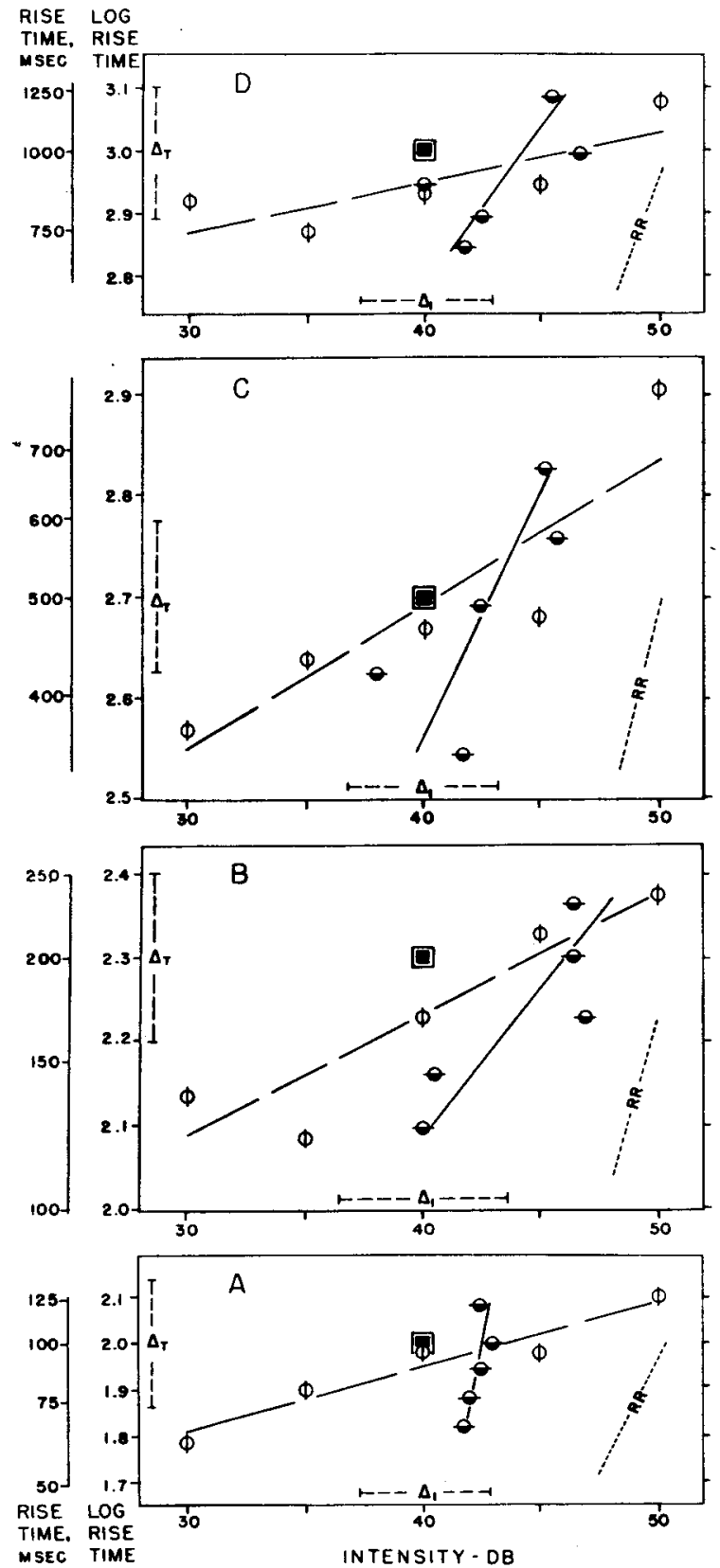

Figure 1. Regression effect in Sheldon's equal-onset contours for vibrotactile stimuli. In each section, the standard which was being matched is shown by the square symbol, $\square$. All standards were $40 \mathrm{~dB}$ SL, but their rise times were $100,200,500$, and $1.000 \mathrm{msec}$ in Panels A through D, respectively. Matches by the method of risetime adjustment are shown as $\phi$. Matches by the method of intensity adjustment are shown as - . Lines marked $\Delta_{T}$ or $\Delta_{I}$ show the size of average test-retest differences. Lines marked $R R$ indicate the slope [but not the position] that regression lines would have if subjects always set the variable stimulus to the same rise rate.

two experiments, but the data obtained by the two methods with a particular standard can be compared more adequately if they are plotted together on the same grid, as has been done in Figure 1 .
The figure is in four sections. Each section shows the contours obtained with one of the four standards cited above. Each section is a $\log -\log$ plot, $\log$ rise time vs. intensity in decibels. The intensity scale is of the same linear extent for all four plots, but the scales of log rise time are of varying extent per log unit from plot to plot. The degree of expansion of each log rise-time scale has been adjusted so that within each plot the two scales are "standardized"- - that is, so that the variability of the subjects' judgments by the method of rise-time adjustment is represented by the same linear distance as the variability of their judgments by the method of intensity adjustment.

The variability data which Sheldon had tabulated were in the form of test-retest differences for each subject and each matching task, every subject having made two matching judgments for each standardvariable combination by each adjustment method. The writer averaged these test-retest differences over all observations made by all the subjects with a given matching method and a particular standard. The average so obtained was taken as a gross within-subject variability index for all matches by that method to that standard. ${ }^{2}$ These average test-retest differences became the basis for adjusting the stimulus scales in Figure 1 for equal variability of judgment and are shown by line segments marked $\Delta_{\mathrm{T}}$ and $\Delta_{\mathrm{I}}$ in each plot.

The data points in each plot are the group medians reported by Sheldon. For want of a convenient and objective assessment of the trends in the data collected by the two methods of adjustment, straight least-squares regression lines have been fitted to the separate sets of data. The dashed lines apply to data that were obtained by the method of rise-time adjustment and that are represented by the $\phi$ symbols, while the solid lines apply to data obtained by the method of intensity adjustment, represented by the symbols. Although the variability of the data indicates that the slopes of the individual regression lines are not so reliable as one might wish, an impressive direction-of-adjustment effect nevertheless appears consistently in all four plots and so can be accepted as reliable.

Examination of Figure 1 leads to the following further observations. First, in Plots A, B, and C, the regression lines are quite symmetrically disposed with respect to the 45-deg line, a condition which may well be related to the adoption of equal variability scales for the stimulus axes. Second, divergence of the regression lines is greatest, and hence the adjustment effect is greatest, in Plot A for the 100-msec standard. Sheldon has reported that short rise-time sensations differed intrinsically from long rise-time sensations, and perhaps this greater effect with the 100 -msec standard arose from this condition. Third, the slopes of the regression lines for the method of intensity adjustment resemble slopes expected if the subjects had been selecting variable stimuli which were of the same rise 
rate (see the line segments labeled RR). Fourth, the divergence of the regression lines for Sheldon's data exceeds that typically observed in experiments on scale matching and cross-modality matching (see, e.g., Indow \& Stevens, 1966; Stevens \& Greenbaum, 1966; Wanchura \& Dawson, 1974).

To one who expects that an experiment like Sheldon's should disclose THE equal response contour with respect to a given standard, these results are disheartening. They imply that equal response contours determined by only a single method or condition of adjustment may be of questionable merit. They also demonstrate that the effect of direction of adjustment on equal response contours can be large enough that attempts to derive some "average" regression line would fail because of uncertainty over how to weigh the separate sets of data.

\section{ERRORS IN SCALE-MATCHING EXPERIMENTS VS. ERRORS IN STUDIES OF EQUAL RESPONSE CONTOURS}

Although Sheldon's experiment has been presented in relation to judgment effects previously observed in scale-matching experiments [e.g.. matching tone loudness and noise loudness. Stevens (1955); matching saturation of one color and saturation of another, Indow de Stevens (1966): matching sound duration and light duration. Stevens \& Greenbaum (1966)], it is to be noted that the regression effect in these studies is conceptually different from that found in Sheldon's case. In scale matching, say in the loudness case, successive matches for loudness are made at different loudness levels (confounded with intensity levels) and the regression is toward some grossly defined mid-intensity-i.e., overall, the subject makes his matches using too narrow a range of intensities with the stimulus he is adjusting. In Sheldon's case, successive matches were made to standards of differing surge, but there is no indication, from a comparison across the plots in Figure 1, of regression toward some mid-surge level delined by the range of standards. Rather, regression occurred within the data for each of the separate standards: the range of rise times (or intensities) which the subjects used in their matches to each standard was narrower than expected. Sheldon comments on the systematic errors that are associated with the displacement of the regression lines from the standard in most cases, but after due allowance for these, one observes that what the subjects did was to narrow the range of their rise-time (or intensity) settings around the rise time (or intensity) of the standard for each contour. In other words, the reference level for potential centering effects in experiments on equal-response contours is the level that the just presented standard occupies on the stimulus dimension that the subject is adjusting.

shifting our level of description, we may say that regression like that observed in Figure 1 would not be expected if subjects were able fully to maintain their criterion of judgment as they shifted from one adjustment method to the other. Sheldon's subjects were making matches that Stevens (1934) once described by the term "trans-dimensional." They were matching on a perceptual dimension or attribute which depends jointly on two independent stimulus dimensions, but not predominantly on either of these stimulus dimensions alone. Stevens (1934) noted, with subjects jud ging auditory volume, that the criterion for such matches tends to be unstable and subject to change. Sheldon's data give further illustration to this point, showing that the criterion can be influenced markedly by the stimulus dimension which the subject happens to be manipulating in order to achieve his match. ${ }^{3}$

\section{NOISE OR SYSTEMATIC ERROR?}

In the foregoing, effects such as Sheldon's are interpreted as associated with judgment bias and systematic errors, not with unreliability or trial-to-trial variability of judgment. It seems reasonable that we call this phenomenon a regression effect, going along with Stevens (1961) in his use of this term for corresponding effects in other experiments that involve matching judgments. In so doing, however, it is important to recognize that the regression in question derives from bias and is not to be confused with regression toward the mean in a correlational or scatterplot sense where the source of the regression is random error. Avoiding this confusion may not always be easy. as Stevens and Greenbaum (1966) shave thenselves demonstrated by their occasional lapses into correlational thinking: "The regression effect presumably occurs whenever the results of the matching judgments yield less than a perfect correlation." And again, "As a general principle, two regression lines are produced whenever the correlation between two variables is less than perfect." And, "Many factors may contribute to the regression tendency, including, ultimately, the irreducible noise that goes with any attempt to measure any empirical value."

The general case for bias as the factor in Sheldon's data can be stated simply. Each of the regression lines concerns a separate univariate situation-the prediction of values on the adjusted stimulus dimcnsion from fixed and well-quantified levels that the experimenter had set on the other stimulus dimension. For such a problem, there is no issue of regression tow ard the mean. the latter being involved only in bivariate problems. Rather, each observed regression slope is an unbiased estimate of the true slope for that standard and method of adjustment. Stevens" regression effect is to be understood in this salme waty (see Cross, 1973). 


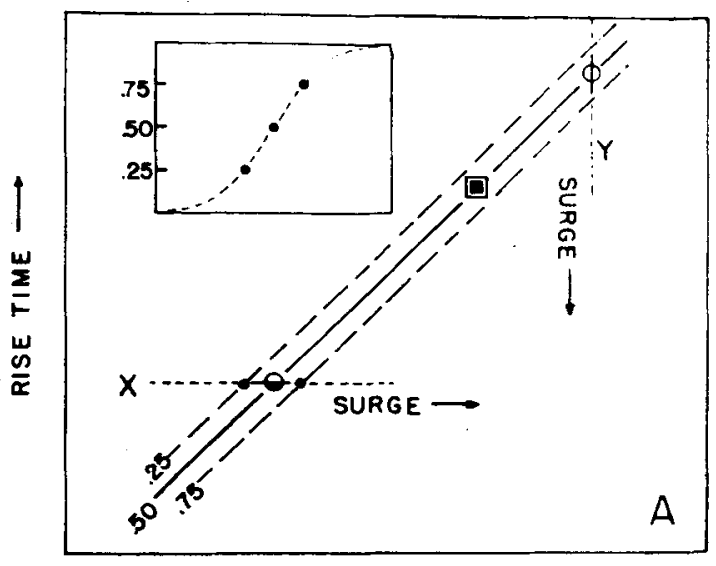

INTENSITY $\rightarrow$

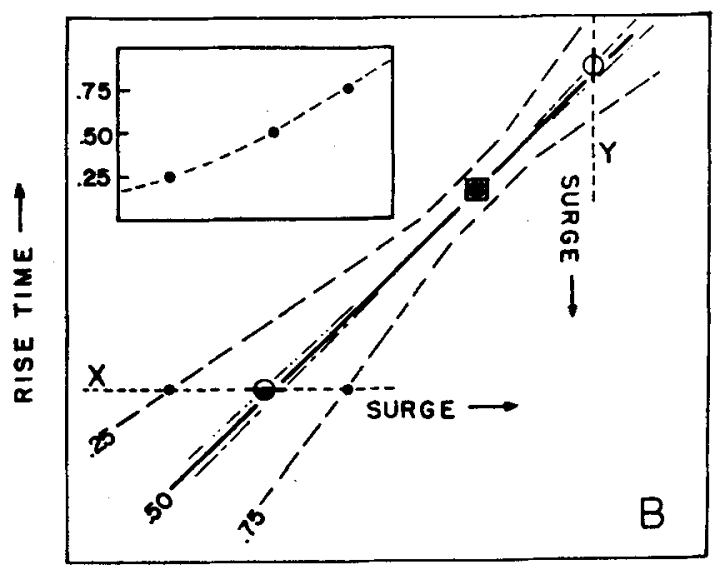

INTENSITY $\longrightarrow$

Figure 2. Probability contours and heteroscedasticity. In A, homoscedasticity is represented, and under the model assumed in the text there is no regression effect. In B, heteroscedasticity produces a small "reverse regression effect." In both $A$ and $B$, the small insert panel in the upper left represents the psychometric function for the range of stimulus conditions along the line $X$ through the intensity:rise-time domain. The small arrows indicate the direction of increasing values of intensity, rise time, and surge.

It is necessary for the present discussion, however, to check out one further condition. Judgments at different points along equal response contours are known to be heteroscedastic. The variability of matching responses or of differential judgments increases as the variable stimulus departs more and more from the standard. This holds for judgments made by the same subject (e.g., see Kingsbury, 1927; Sheldon, 1973) as well as for subject-to-subject differences in mean judgment (e.g., see Robinson \& Dadson. 1956; Thomas, 1949). In fact, very wide differences between variable and standard eventually make transdimensional matching judgments impossible (Sheldon, 1973; Thomas, 1949). We should therefore verify that this particular form of heteroscasdicity is not itself involved in the regression found in Sheldon's data. The following analysis clarifies this point.
Consider the "true" equal response contour for a givensubject to be delined as a contour which divides a ino-dimemsional stimulus domain into two regions in such at manner that for every point in one of them the probability of his judging the variable stimulus to have more than the standard does of the perceptual property under investigation is greater than .50. while for every point in the other that probability is less than .50) By delinitions which parallel this one, we can identify still wher conlours, saly the .25 contour which provides regions where the probabilities are greater and less than .25 . the .75 contour, etc.

Suppose now a limited and ideally simplified set of conditions-that the equal response contour is a straight line on a standardized plot. and that homoscedasticity applies in both stimulus dimensions at all points along the contour. Under these conditions, the $.25 \mathrm{and} .75$ contours must be parallel to the .50 contour. See Figure 2A. If the .25 and .75 contours and all other complementary contours are symmetrically disposed with respect to the .50 contour, then barring judgment bias as the subject scans the variable stimulus conditions along a series such as that represented by the line $X$ (or the line $Y$ ) in Figure $2 \mathrm{~A}$. his mean match should agree with the median of his psychometric function for the stimulus set $X$ (or $Y$ ), and the means for both methods of adjustment should all fill on the .50 contour, as shown in the figure. Hence, for these conditions with no adjustment bias, both methods of adjustment would establish an identical contour, namely the equal response contour as defined above, and they would do so regardless of whether the variability of the psychometric function was large or small.

The heteroscedasticity cited above, however, implies for our subject a set of probability contours something like that shown in Figure 2B. Here the psychometric functions along the sequence of stimulus levels which the subject scans in his adjustments must all be skewed. Under these circumstances, it appears that the subject's mean adjustment should deviate somewhat fron the PSE obtained from the .50 point of the psychometric function and that this deviation will be toward the longer tail of the skewed ogive. This condition would, in fact, lead to two intersecting regression lines, drawn at only a small angle of divergence in the figure, but what we quickly observe is that these lines cross in the reverse manner from that observed in the Sheldon experiment and in the various matching experiments reviewed by Stevens and Greenbaum.

There are no grounds, then, for discounting systematic error as the basis for the regression effect in Sheldon's data.

\section{THE THOMAS STUDY OF EQUAL VOLUME CONTOURS}

Among studies of equal-response contours over 
bi-dimensional stimulus domains, there is, to the writer's knowledge, only one in addition to Sheldon's which has collected data by giving the subject control over first one stimulus dimension and then the other. This is the study by Thomas (1949) of equal-volume contours over the auditory frequency:intensity. domain. A comparison of the two studies is intormative.

Thomas reports median data on five subjects. Some were trained on the frequency adjustment method first, some on intensity adjustment, but eventually all used both methods. All reported that it was easier to make their volume matches by the method of frequency adjustment. Shcldon's subjects, incidentally, also had a method preference: they found the rise-time adjustment method subjectively easier.

From his graphs, one must conclude that Thomas' equal volume contours exhibit little if any overall difference in slope associated with direction of adjustment. Contours for the two methods were much alike over much of their range. Thomas did observe, however, that the contours for the method of intensity adjustment tended to be sigmoid in shape in comparison with the contours for frequency adjustment. This would imply a divergence of the contours in any pair at their upper and lower limits. Such divergence was most obvious over the low-lrequency:low-intensity portions of all three pairs of contours obtained for standards of $1.000 \mathrm{~Hz}$. The divergence here was consistent and progressive, and was in lact in the direction expected on the basis of bias foward the level of the standard on the adjusted stimulus dimension.

Taken together, the results reported by Sheldon and Thomals suggest the hypothesis that in studies of equal response contours using the method of adjustment, the probability of bias toward the level of the standard will increase with the difticulty of the matching task. Thomas clearly arranged the easier matching situation. He was able to use a rapid stimulus sequence with all stimuli of short rise time and duration, which Sheldon could not do, and he presented the standard and variable in continuous alternation, a condition which regularly favors good discrimination. ${ }^{4}$ These differences probably account for much of the overall difference in regression between the experiments. Further. increased difficulty near the limits of Thomas' contours for $1.000 \mathrm{~Hz}$ standards could have been the basis of the developing bias observed there.

It is of interest to note that Stevens and Greenbaum (1966) also considered situational difficulty important in accounting for differences in the extent of regression effects in cross-modality matching.

\section{SUMMARY AND CONCLUSIONS}

Sheldon's data on equal onset contours for vibrotactile stimuli display a matked regression effect.
This regression is due to differential bias, not unreliability or random error, and is not to be confused with statistical regression toward the mean. It resembles the effect discussed by Stevens and others with respect to data from scale-matching and other experiments, but is dependent upon the character of the just presented standard rather than upon the overall stimulus range. Both of these regression effects appear to increase with the difficulty of the matching task.

These regression effects arise when the method of adjustment is used, and can be avoided in the determination of equal response contours or indifference contours through the use of alternative methods [e.g., see Campbell \& Masterson (1969), in their study of aversive stimuli; Kappauf, Burright, \& DeMarco (1963), in their study of iso-hedonic contours in taste; Kingsbury (1927), in his study of equal loudness contours). If, because of its special convenience, the method of adjustment is to be explored for obtaining such contours, then the experiments of Sheldon and Thomas suggest that preliminary data should always be collected using adjustments in both stimulus dimensions so that the regression effect can be assessed. In this testing, it would seen desirable to have the subject use the two methods of adjustment on alternate trials in order to reduce systematic effects of possible long-term shifts in the criterion of match to the standard. Should this preliminary testing disclose a regression effect that cannot be held within acceptably narrow limits, the problem can be averted by shifting to some other method of data collection. ${ }^{5}$

\section{REFERENCES}

Campbell, B. A., \& Masterson, F. A. Psychophysics of punishment. In B. A. Campbell and R. M. Church (Eds), Parnishment and aversive behavior. New York: AppletonCentury-Crofts. 1969. Pp. 3-42.

Cross, D. V. Sequential dependencies and regression in psychophysical judgments. Perception \& Psychophysics, 1973, 14. \$47-552.

Hollingworth, H. L. The central tendency in judgment. Journal of Philosophy. Psychology and Scientific Methods. 1910. 7. 461.469 .

Innow. T. \& Srevens, S. S. Scaling of saturation and hue. Porception \& Psychophysics, 1966. 1.253-271.

Kappauf, W. E. The application of conditioning methods to the study of discrimination and the measurement of differential thresholds in animals. Jourmal of Psychology, 1943, 15. 129-135.

Kappauf, W. E., Burright, R. G., \& Demarco, W. Sucrosequinine mixtures which are isohedonic for the rat. Journal of Comparative and Physiological Psychology' 1963, 56. 138-143.

KingsBury. B. A. A direct comparison of the loudness of pure tones. Physical Review (Ser. 2), 1927, 29, 588-600.

Robinson, D. W., \& Dadson, R. S. A re-determination of the equal-loudness relations for pure tones. British Joumal of Applied Physics, 1956, 7, 166-181.

Sheidon. P. E. Equal-onset contours of vibrotacritc stimuli. Perception \& Psychophysics, 1973, 13, 403-407.

Stevens. S. S. The whime and intensity of tones. Americtan Jounal of Psycholog!' 19.34. 46. 397-408. 
Stevens. S. S. The measurement of loudness. Journal of the Acoustical Society of America, 1955, 27, 815-829.

Stevens. S. S. The psychophysics of sensory function. In W. A. Rosenblith (Ed.). Sensory communication. Cambridge. Mass: M.I.T. Press. 1961. Pp. 1-33.

STEVENS. S. S. On predicting exponents for cross-modality matches. Perception \& Psychophysics. 1969. 6. 251-256.

Stevens. S. S.. \& Greenbaum, H. B. Regression effect in psychophysical judgment. Perception \& Psychophysics, 1966, 1. 439.446.

Thомas, G. J. Equal-volume judgments of tones. American Journal of Psychology. 1949. 62. 182-201.

Wanschura, R. G., \& Dawson, W. E. Regression effect and individual power functions over sessions. Journal of Experimental Psychology. 1974, 102, 806-812.

\section{NOTES}

1. Personal communication. The writer extends his thanks to Dr. Sheldon for making his original data available.

2. This averaging is a relatively crude procedure in view of the knewn increase in varjability of matching judgments as the "lixed dimension" of the variable stimulus differs more and more from the kevel of the standard on that dimension. and in view of Shekdon's repont of great inconsistency of judgment when rise-time adjustments at intensity levels of 25 and $55 \mathrm{~dB}$ were attempted. Nonetheless. the obtained average variabilities were useful in the present attempt to approximate a standardized plot.

3. It was noted above that Sheldon's data were collected in iwo temporally distinct experiments, all of the data by the method of rise-time adjustment having been collected first. That this admittedly confounding factor may in itself have been responsible for the marked regression or criterion shift is unlikely in wiew of the fact that all subjects apparently shifted together: the regression slopes for the subjects individually, as reported by Sheldon, were similar in each of the two experiments.

4. Such a sequence is also recognized to simplify discrimination training in animals. See Kappauf (1943).

5. For an early evaluation of the method of constant stimuli, see Stevens (1934).

(Received for publication November 6, 1974: accepted November 18, 1974.) 